\section{A) Check for updates}

Cite this: Nanoscale, 2020, 12, 23234

\title{
A glycoconjugate-based gold nanoparticle approach for the targeted treatment of Pseudomonas aeruginosa biofilms $\dagger$
}

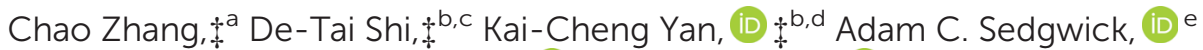 \\ Guo-Rong Chen, ${ }^{b}$ Xiao-Peng He, (D) ${ }^{b}$ Tony D. James, (D) ${ }^{d}$ Bing Ye, ${ }^{* a}$ Xi-Le Hu*b and \\ Daijie Chen ${ }^{\star}$
}

In this study, "core-shell" gold nanoparticles (AuNPs) have been functionalised using a simple one-pot approach to form fucose-based glycoconjugate AuNPs (Fuc-AuNPs) and galactose-based glycoconjugate AuNPs (Gal-AuNPs), respectively. Owing to the selective carbohydrate-based recognition of the key virulence factors of $P$. aeruginosa, LecB (fucose-specific lectin)/LecA (galactose-specific lectin), FucAuNPs and Gal-AuNPs-based imaging and therapeutic strategies were evaluated towards $P$. aeruginosa. Both Fuc-AuNPs and Gal-AuNPs were non-covalently loaded with the fluorophore dicyanomethylene $4 \mathrm{H}$-pyran (DCM) to afford two highly selective fluorescence imaging agents for the visualisation of P. aeruginosa. The loading of Fuc-AuNPs and Gal-AuNPs with the known antibiotic Ceftazidime (CAZ) exhibited an enhanced therapeutic effect, illustrating the significance of this targeted drug delivery strategy. Exploiting the phototherapeutic properties of AuNPs, photoirradiation (600 nm) of Fuc-AuNP@CAZ/ Gal-AuNPaCAZ provided both photothermal and photodynamic therapeutic (PTT/PDT) effects, which facilitated the release of CAZ. Fuc-AuNP@CAZ and Gal-AuNP@CAZ were shown to be effective photo/ chemotherapeutics resulting in almost complete eradication of $P$. aeruginosa biofilms formed on clinically relevant surfaces (glass slides and steel surface).

Received 18th July 2020 ,

Accepted 7th November 2020

DOI: $10.1039 /$ d0nr05365a

rsc.li/nanoscale and cystic fibrosis (CF) patients. ${ }^{3,4}$ Moreover, $P$. aeruginosa has the ability to form biofilms on mucosal surfaces and medical implant devices, ${ }^{1}$ which provide favourable and protective conditions for bacterial survival against host defence mechanisms and aggressive antibiotic treatment. ${ }^{1,5,6}$ As a direct result, this has led to a continued increase in resistant and multidrugresistant (MDR) P. aeruginosa and a restriction in treatment options. ${ }^{7}$ Therefore, $P$. aeruginosa is now considered as a major public health threat. ${ }^{8-10}$

Rapid advancements in material-based sciences has led to the identification of a range of drug delivery platforms with inherent diagnostic/therapeutic properties (i.e. photodynamic therapy/photothermal therapy (PDT/PTT)). ${ }^{11-15}$ These systems have been demonstrated as effective anticancer and antimicrobial agents that can be activated by single or dual excitation wavelengths (e.g. $660 \mathrm{~nm}$ and $808 \mathrm{~nm}) .{ }^{12,14,16-22}$ Researchers have continued to explore these platforms by incorporating additional antimicrobial metals such as copper $(\mathrm{Cu})$ and silver $(\mathrm{Ag}) \cdot{ }^{23-27}$ Within the field of material sciences, gold nanoparticles (AuNPs) are of particular interest owing to their unique multifunctional and biocompatible properties. ${ }^{11,28}$ The high surface area-to-volume ratio and ease of functionalisation of AuNPs has provided the capability to incorporate 
therapeutics or targeting ligand units (i.e. peptides, carbohydrates and genetic material) onto the surface of AuNPs through covalent/non-covalent interactions. ${ }^{28-31}$ As a result, AuNPs provide an attractive platform for the effective delivery of functional molecules through active and passive targeting. ${ }^{32}$ In conjunction, with their ability to selectively deliver therapeutic agents, ${ }^{30,33,34}$ AuNPs have been shown as effective phototherapeutics with the production of cytotoxic ROS (PDT) and/or heat (PTT) ${ }^{35-38}$ Despite these promising attributes, the application of AuNPs have been mostly directed towards the development of anticancer agents with minimal attention on their use as antimicrobials. ${ }^{14,39}$ Therefore, we envisioned tailoring the design of AuNPs to target specific bacteria would allow the controlled and selective delivery of antibiotics. Moreover, in combination with PTT/PDT, we believed this would afford an effective antimicrobial platform with minimal off-target toxicities ${ }^{30}$ and could reduce the potential to develop MDR bacteria.

A key step in the pathogenesis of $P$. aeruginosa is the adhesion of bacterial cells to human tissue, ${ }^{40}$ which is believed to occur through the targeted recognition of mammalian glycoproteins by virulence factors such as lectins (LecA and LecB). ${ }^{41}$ Exploiting the selective carbohydrate-based recognition of LecA (galactose specific) and LecB (fucose specific), we have developed two glycoconjugate-based AuNPs which were coated with the monosaccharides fucose (Fuc-AuNPs) and galactose (Gal-AuNPs), respectively (Scheme 1). NIR fluoro-

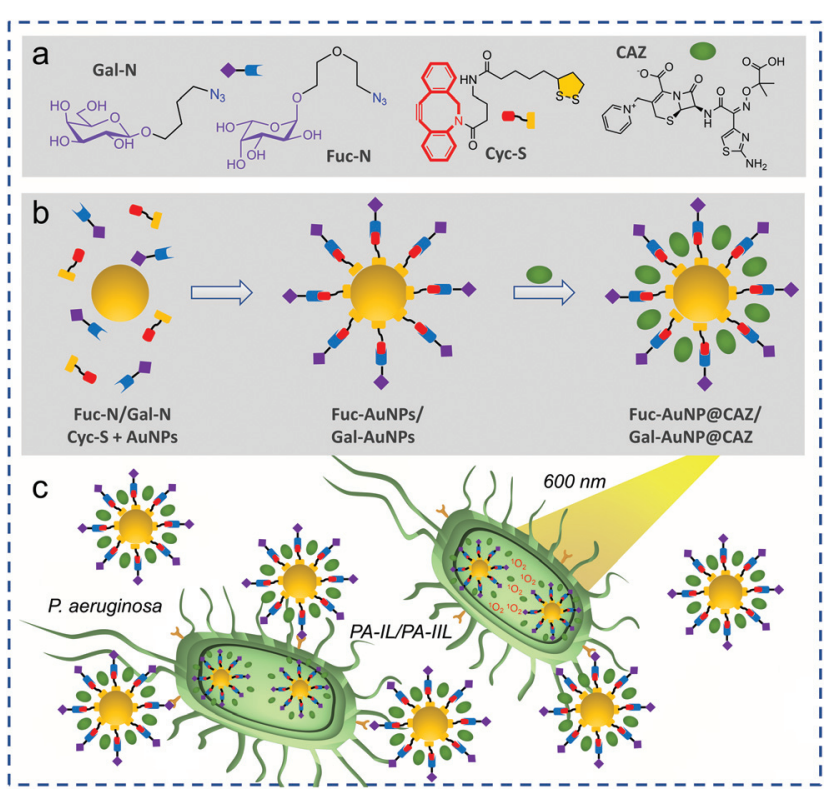

Scheme 1 (a) Key building blocks for the construction of Fuc-AuNPs and Gal-AuNPs: carbohydrate functionalised azide (Fuc-N and Gal-N) and cyclooctyne tailed disulfide ring (Cyc-S). Antibiotic as co-loaded molecular cargo: Ceftazidime (CAZ). (b) Schematic illustration of the one-pot self-assembly procedure and co-loading of CAZ to form FucAuNP@CAZ/Gal-AuNP@CAZ. (c) Schematic illustration of the targeted lectin (LecB/LecA, also called PA-IIL/PA-IL) approach of Fuc-AuNP@CAZ/ Gal-AuNP@CAZ to selectively enter $P$. aeruginosa to simultaneously release CAZ and generate ROS/heat upon photoirradiation (600 nm). phore, dicyanomethylene- $4 H$-pyran (DCM $)^{42}$ loaded AuNPs (Fuc-AuNP@DCM and Gal-AuNP@DCM) afforded two highly selective imaging agents for the visualisation of $P$. aeruginosa. In addition, this result suggests binding with $P$. aeruginosa facilitates the release of the molecular cargo from the AuNPs. As a result, both Fuc-AuNPs and Gal-AuNPs were subsequently loaded with the antibiotic Ceftazidime (CAZ), ${ }^{43}$ to afford the dual photo/chemotherapeutics, Fuc-AuNP@CAZ and GalAuNP@CAZ. This targeted lectin approach improved the therapeutic efficacy of CAZ and in combination with photoirradiation (600 nm), Fuc-AuNP@CAZ and Gal-AuNP@CAZ exhibited the effective destruction of the established $P$. aeruginosa biofilms on clinically relevant surfaces - glass slides and steel surfaces (Scheme 1).

Copper-catalysed alkyne-azide cycloaddition (CuAAC) chemistry is routinely used for the synthesis of glycoconjugates. ${ }^{44}$ Unfortunately, the use of $\mathrm{Cu}(\mathrm{I})$ ions for CuAAC chemistry can prove problematic for certain reaction substrates. ${ }^{45}$ To overcome this limitation, strain-promoted "click" chemistry has been developed. In brief, a strained cyclooctyne unit covalently linked to a disulfide ring (Cyc-S) was used to form a self-assembled monolayer (SAM) on the surface of AuNPs. ${ }^{46}$ Subsequent "click" chemistry between AuNP-Cyc-S (on the gold surface) and azide Fuc-N (fucosyl) or Gal-N (galactosyl) afforded the desired Fuc-AuNPs and Gal-AuNPs (see ESI $\dagger$ for the one-pot procedure). This was confirmed by a clear signal enhancement in the Raman spectrum (see ESI - Fig. S1†). ${ }^{47,48}$ Moreover, zeta potential measurements demonstrated good aqueous stability for Fuc-AuNPs and Gal-AuNPs (see ESI Fig. S1†).

With both Fuc-AuNPs and Gal-AuNPs in hand, the lectin binding properties of each AuNP was then evaluated. The addition of UEA-1 (Ulex European Agglutinin 1, fucose-specific lectin) to an aqueous solution of Fuc-AuNPs (0.2 nM) led to the broadening as well as a bathochromic shift of the UV-Vis absorption at $600 \mathrm{~nm}$ (Fig. 1a). This was attributed to aggregation of the AuNPs. ${ }^{49,50}$ Minimal changes to the UV-vis absorption of Fuc-AuNPs were seen with the addition of other lectins/proteins (Fig. 1b), which was indicative of fucose selective binding. Similarly, the addition of PNA (Peanut Agglutinin, galactose-specific lectin) to a solution of GalAuNPs (0.2 nM) led to a gradual broadening as well as bathochromic shift of the UV-vis absorption at $600 \mathrm{~nm}$ (Fig. 1c and d). Again, minimal changes were observed when in the presence of other lectins/proteins. In addition, both Fuc-AuNPs and Gal-AuNPs demonstrated a high sensitivity for each corresponding lectin with low limit of detection (LoD) FucAuNPs - UEA-1 = 13.8 nM/Gal-AuNPs - PNA = 4.9 nM (see ESI - Fig. S2†).

HR-TEM (High-Resolution Transmission Electron Microscope) images and Dynamic Light Scattering (DLS) analysis revealed no obvious differences between unmodified AuNPs and Fuc-AuNPs/Gal-AuNPs (Fig. 2 and Fig. S3 $\dagger$ ). However, in the presence of their corresponding lectins, both Fuc-AuNPs and Gal-AuNPs were shown to form large clusters, which was indicative of lectin binding and lectin-mediated 
a

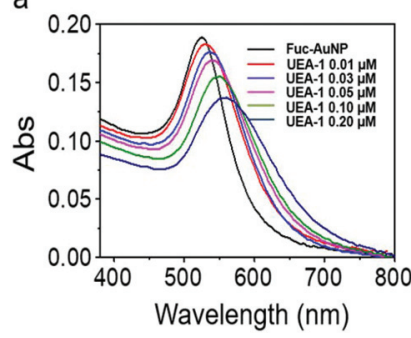

C

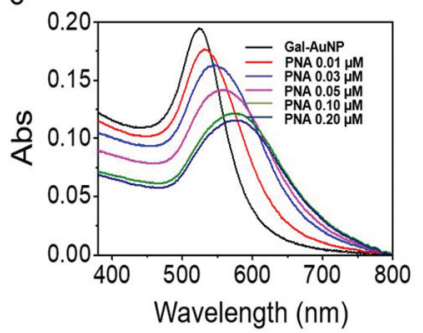

b

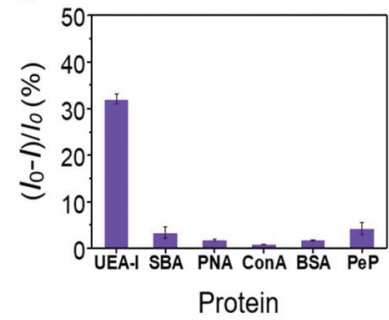

d

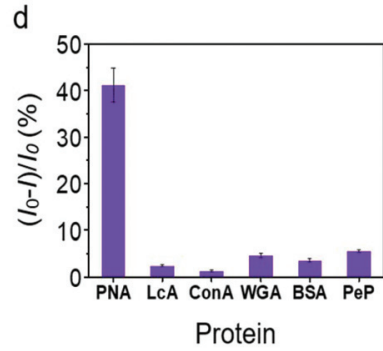

Fig. 1 (a) UV-vis spectra of Fuc-AuNPs (0.2 nM) with increasing concentrations of Ulex European Agglutinin 1 (UEA-1: 0.01-0.2 $\mu \mathrm{M}$ ) in Tris$\mathrm{HCl}$ buffer. (b) Change in UV-vis absorption at $525 \mathrm{~nm}$ of Fuc-AuNPs $(0.2 \mathrm{nM})$ in the presence of UEA-1, SBA (Soybean Agglutinin), PNA, Con A (Conconvalin A), BSA (Bovine Serum Albumin), PeP (Pepsin) (protein concentrations: $0.05 \mu \mathrm{M})$. (c) UV-Vis spectra of Gal-AuNPs $(0.2 \mathrm{nM})$ with increasing concentrations of Peanut Agglutinin (PNA: 0.01-0.2 $\mu \mathrm{M}$ ) in Tris- $\mathrm{HCl}$ buffer. (d) Change in UV-Vis absorption at $525 \mathrm{~nm}$ of GalAuNPs $(0.2 \mathrm{nM})$ in the presence of PNA, LcA (Lens culinaris Lectin), Con A (Conconvalin A), WGA (Wheat Germ Agglutinin), BSA (Bovine Serum Albumin), PeP (Pepsin) (Protein concentrations: $0.05 \mu \mathrm{M})$.

a

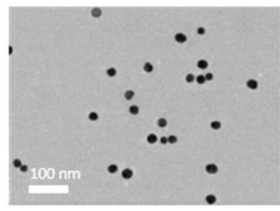

b

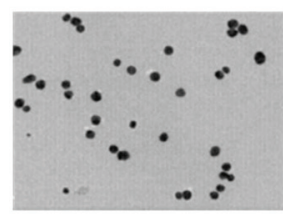

C

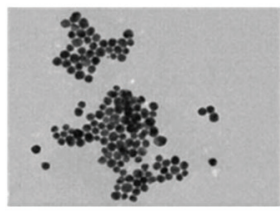

d

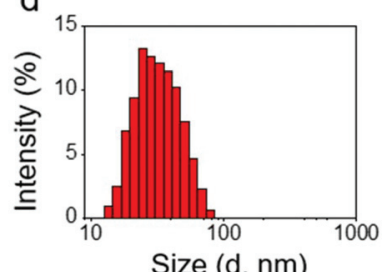

e

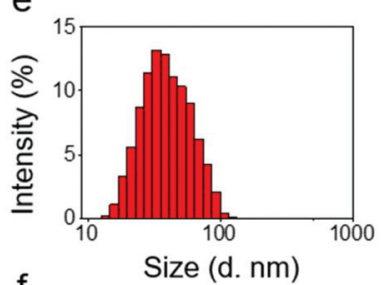

$f$

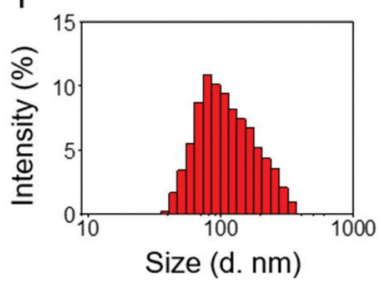

Fig. 2 High-Resolution Transmission Electron Microscope (HR-TEM) images: (a) AuNPs (0.02 nM) (b) Fuc-AuNPs (0.02 nM) (c) Fuc-AuNPs $(0.02 \mathrm{nM})+$ UEA-1 $(0.02 \mu \mathrm{M})$. Dynamic Light Scattering (DLS): (d) AuNPs $(0.2 \mathrm{nM})$ (e) Fuc-AuNPs (0.2 nM) (f) Fuc-AuNPs (0.2 nM) + UEA-1 $(0.05 \mu \mathrm{M})$.

agglutination (Fig. 2 and see ESI - Fig. S3 and S4 $\dagger$ ). ${ }^{51}$ In addition, this further supported the lectin-mediated changes observed in the UV-Vis absorption of Fuc-AuNPs and GalAuNPs shown in Fig. $1 \mathrm{a}$ and c.

Upon identifying the lectin-binding properties of FucAuNPs and Gal-AuNPs, the drug loading and phototherapeutic properties were then evaluated. Fuc-AuNPs or Gal-AuNPs (0.8 nM) were mixed in an aqueous solution containing CAZ for $4 \mathrm{~h}$. Subsequent centrifugation (10000 rpm) and washing (Tris-HCl buffer) afforded both Fuc-AuNP@CAZ and GalAuNP@CAZ. Both the loading of CAZ and corresponding AuNP@CAZ nanoparticle stability was confirmed by Raman spectroscopy and zeta potential measurements, respectively (see ESI - Fig. S1 $\dagger$ ). The non-covalent wrapping interactions ${ }^{52}$ between CAZ and Fuc-AuNPs and Gal-AuNPs were found to be stable over several days with no clear changes to both zeta potentials and the particle sizes (see ESI - Fig. S5†). Next, the phototherapeutic (PDT and PTT) properties of these systems were evaluated. $600 \mathrm{~nm}$ was chosen as the excitation wavelength for PDT/PTT experiments as this is the maximum absorption wavelength of Fuc-AuNPs/Gal-AuNPs. The known ROS fluorescent probe dihydrorhodamine was used to confirm ROS production upon light irradiation (600 nm, $1 \mathrm{~W} \mathrm{~cm} \mathrm{~cm}^{-2}$ ). The ROS colorimetric probe tetramethylbenzidine (TMB) was used to confirm the production of ROS using UV-Vis spectroscopy. ${ }^{53}$ Both Fuc-AuNPs and Gal-AuNPs were shown to produce ROS under light irradiation. No significant changes to ROS production were observed in the presence of lectins or by varying the $\mathrm{pH}$ and temperature (see ESI - Fig. S6 $\dagger$ ). Previous reports have shown the main ROS produced is ${ }^{1} \mathrm{O}_{2} \cdot{ }^{33,54}$ Interestingly, the light irradiation of UEA-1, AuNPs and FucAuNP@CAZ displayed minimal PTT effects, however, upon lectin-binding a dramatic temperature increase was observed (see ESI - Fig. S7†). This observation is believed to be the result of the lectin-mediated formation of Fuc-AuNP@CAZ clusters allowing the generated heat to be localized, which enables an efficient increase in temperature. In contrast, when simple AuNPs are dispersed in solution, this results in a dissipation of the heat resulting in a poor PTT effect being observed. For Fuc-AuNPs and Gal-AuNPs in the presence of their corresponding lectins, a PTT conversion efficiency of $26.2 \%$ and $25.7 \%$ was observed respectively (see ESI Fig. S8†). Fuc-AuNP@CAZ binding to P. aeruginosa (with LecA expression similar to UEA-1) was also shown to have a good PTT effect (see ESI - Fig. S9†). Demonstrating a potential mechanism of action, Fuc-AuNP@CAZ and Gal-AuNP@CAZ systems were shown to release CAZ gradually in the presence of either light irradiation or lectins (see ESI - Fig. S10 and $\mathrm{S} 11 \dagger)$. No unwanted CAZ release was observed in their absence, which is in accordance with the stability data for FucAuNP@CAZ and Gal-AuNP@CAZ systems (see ESI - Fig. S5 $\dagger$ ). According to these results, it is believed that the combined PDT and PTT effect will not only contribute to the overall therapeutic efficacy of CAZ towards $P$. aeruginosa, but also act as "triggers" to accelerate the release of co-loaded molecular cargos. 
To confirm the LecB/LecA specificity and $P$. aeruginosa uptake of molecular cargos loaded on these glycoconjugatebased AuNPs, the fluorophore dicyanomethylene $4 H$-pyran (DCM) (see ESI $\dagger$ for chemical structure) ${ }^{42}$ was loaded onto FucAuNPs/Gal-AuNPs. In addition, this study was used to illustrate lectin binding from $P$. aeruginosa facilitates the release of the molecular cargo. Using a similar loading protocol, Fuc-AuNPs or Gal-AuNPs (0.8 nM) were mixed in an aqueous solution containing DCM $(10 \mu \mathrm{M})$. Subsequent centrifugation $(10000 \mathrm{rpm})$ and washing (Tris-HCl buffer) afforded both Fuc-AuNP@DCM and Gal-AuNP@DCM. As seen in Fig. 3, P. aeruginosa (ATCC 27853) treated with either Fuc-AuNP@DCM or Gal-AuNP@DCM displayed an obvious fluorescence emission signal, which suggested the uptake of the DCM. Confirming the LecB and LecA-mediated uptake of the NIR fluorophore, $P$. aeruginosa was pre-treated with either free L-fucose or D-galactose $(10 \mathrm{mM})$. This led to a marked reduction in fluorescence emission intensity being observed. This was believed to be due to the pre-saturation of the lectin binding sites which prevented the binding and uptake of Fuc-AuNP@DCM/ Gal-AuNP@DCM. Overall, our approach demonstrates the targeted release of molecular cargos in P. aeruginosa.

Next, we turned our attention towards the evaluation of the antibacterial efficacy of each glycoconjugate-based AuNPs with and without light irradiation (30 $\left.\mathrm{min}, 600 \mathrm{~nm}, 1 \mathrm{~W} \mathrm{~cm}^{-2}\right)$. In this study, $P$. aeruginosa (ATCC 27853) and drug-resistant $P$. aeruginosa (ATCC BAA2110) were treated with CAZ only, FucAuNPs, Gal-AuNPs, Fuc-AuNP@CAZ, and Gal-AuNP@CAZ, respectively (Fig. 4). The sole use of CAZ $\left(2 \mu \mathrm{g} \mathrm{mL} \mathrm{mL}^{-1}\right)$ resulted in a moderate inhibition with no additional effect seen under light irradiation ( $30 \mathrm{~min}, 600 \mathrm{~nm}, 1 \mathrm{~W} \mathrm{~cm}^{-2}$ ). Fuc-AuNPs and Gal-AuNPs were shown to have no "dark" therapeutic effect, whereas, the light irradiation of Fuc-AuNPs/Gal-AuNPs displayed a modest therapeutic effect with a slightly greater efficacy compared to unmodified AuNPs (see ESI - Fig. S12 $\dagger$ ).

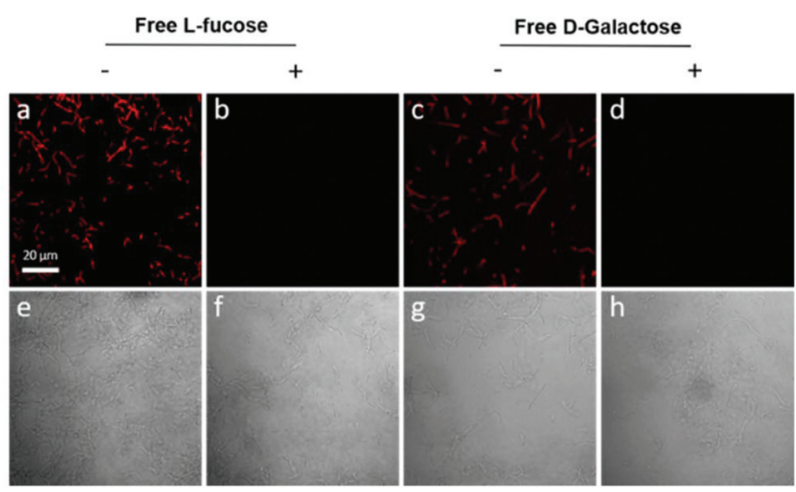

Fig. 3 Confocal Laser-Scanning Microscope (CLSM) images of P. aeruginosa (ATCC 27853): (a) Fuc-AuNP@DCM (0.2 nM and $1 \mu \mathrm{M}$ ) (b) pre-treatment with L-fucose $(10 \mathrm{mM})$ followed by addition of FucAuNPaDCM (0.2 nM and $1 \mu \mathrm{M}$ ) (c) Gal-AuNP@DCM (0.2 nM and $1 \mu \mathrm{M})$ (d) pre-treatment with $D$-galactose $(10 \mathrm{mM})$ followed by addition of GalAuNPaDCM (0.2 nM and $1 \mu M)(e-h)$ bright field images of (a-d).
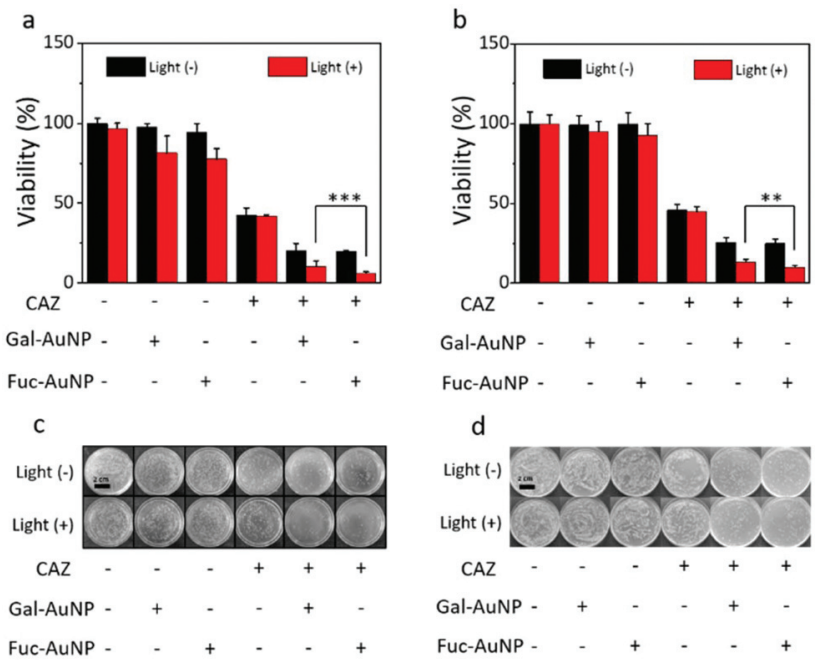$$
\text { d }
$$

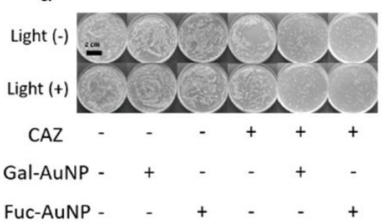

Fig. 4 (a) Relative viabilities of $P$. aeruginosa (ATCC 27853) treated with sterile water (control), Fuc-AuNPs (0.2 nM), Gal-AuNPs (0.2 nM), Ceftazidime (CAZ, $2 \mu \mathrm{g} \mathrm{mL}^{-1}$ ), Fuc-AuNPaCAZ $\left(0.2 \mathrm{nM}\right.$ and $\left.2 \mu \mathrm{g} \mathrm{mL}^{-1}\right)$, and Gal-AuNP@CAZ $\left(0.2 \mathrm{nM}\right.$ and $\left.2 \mu \mathrm{g} \mathrm{mL}^{-1}\right)$ with and without light irradiation (30 $\mathrm{min}, 600 \mathrm{~nm}, 1 \mathrm{~W} \mathrm{~cm}{ }^{-2}$ ). (b) Relative viabilities of drugresistant $P$. aeruginosa (ATCC BAA2110) treated with sterile water (control), Fuc-AuNPs (0.2 nM), Gal-AuNPs (0.2 nM), Ceftazidime (CAZ, $\left.2 \mu \mathrm{g} \mathrm{mL}{ }^{-1}\right)$, Fuc-AuNPaCAZ $\left(0.2 \mathrm{nM}\right.$ and $\left.2 \mu \mathrm{g} \mathrm{mL} \mathrm{m}^{-1}\right)$, and GalAuNPaCAZ $\left(0.2 \mathrm{nM}\right.$ and $\left.2 \mu \mathrm{g} \mathrm{mL} \mathrm{m}^{-1}\right)$ with and without light irradiation (30 min, $600 \mathrm{~nm}, 1 \mathrm{~W} \mathrm{~cm}^{-2}$ ). (c) P. aeruginosa (ATCC 27853) colonies on Luria-Bertani (LB) agar plates treated with the respective systems with and without light irradiation (30 $\mathrm{min}, 600 \mathrm{~nm}, 1 \mathrm{~W} \mathrm{~cm}^{-2}$ ). (d) $P$. aeruginosa (ATCC BAA2110) colonies on Luria-Bertani (LB) agar plates treated with the respective systems with and without light irradiation (30 $\min , 600 \mathrm{~nm}, 1 \mathrm{~W} \mathrm{~cm}{ }^{-2}$ ) $* * * P<0.001 ; * * P<0.01$.

The drug delivery capability of Fuc-AuNPs and Gal-AuNPs were confirmed with an enhanced therapeutic effect seen in comparison to CAZ. Remarkably, Fuc-AuNP@CAZ and Gal-AuNP@CAZ under light irradiation (30 min, $600 \mathrm{~nm}, 1 \mathrm{~W}$ $\mathrm{cm}^{-2}$ ) demonstrated a significant cooperative therapeutic effect with CAZ, which almost resulted in full eradication $(\approx 90 \%$ ) (Fig. 4). This cooperative therapeutic effect was further confirmed using a LIVE/DEAD cell viability assay (see ESI Fig. S13 $\dagger$ ). HR-TEM images were used to demonstrate the mode of action with Fuc-AuNP@CAZ initially interacting on the surface of $P$. aeruginosa and the subsequent light irradiation ( $30 \mathrm{~min}, 600 \mathrm{~nm}, 1 \mathrm{~W} \mathrm{~cm}{ }^{-2}$ ) resulting in a clear change to the morphology of the bacteria (see ESI - Fig. S14 $\dagger$ ). Confirming the selectivity for $P$. aeruginosa, the treatment of E. coli and methicillin-resistant $S$. aureus with Fuc-AuNP@CAZ/ Gal-AuNP@CAZ were shown to have a significantly lower therapeutic outcome (see ESI - Fig. S15†). No toxicity was observed towards both 293T (human renal epithelial) and MDA-MB-231 (human breast cancer) cells (see ESI - Fig. S16†).

To demonstrate the full therapeutic potential of our glycoconjugate-based AuNPs strategy, P. aeruginosa biofilms were established on glass slides and steel surfaces. In these experiments, the performances were evaluated using the LIVE/DEAD biofilm viability assay (PI, dead - yellow and Syto9®, live - 
blue). As shown in the processed 3D images of $P$. aeruginosa biofilms (Fig. 5), the use of just CAZ led to a partial antibiofilm effect, however, when co-loaded with Fuc-AuNPs or Gal-AuNPs, an enhanced antibiofilm effect was observed. Remarkably, light irradiation $\left(600 \mathrm{~nm}, 30 \mathrm{~min}, 1 \mathrm{~W} \mathrm{~cm} \mathrm{~cm}^{-2}\right)$ of FucAuNP@CAZ/Gal-AuNP@CAZ resulted in almost complete biofilm destruction on both glass slides and on clinically relevant steel surfaces (Fig. 5). Overall, this illustrates the potential of Fuc-AuNP@CAZ/Gal-AuNP@CAZ as excellent light and chemo-based antimicrobials for the targeted treatment of $P$. aeruginosa.

In summary, fucose and galactose-based glycoconjugated AuNPs (Fuc-AuNPs and Gal-AuNPs) were developed using a simple one-pot self-assembly procedure. Both Fuc-AuNPs and Gal-AuNPs displayed selective lectin-binding properties as determined by UV-Vis, HR-TEM and DLS analysis. The FucAuNPs and Gal-AuNPs targeting ligands were effective for the
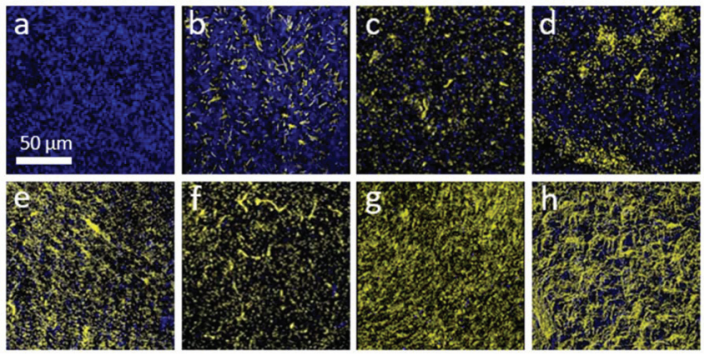

i

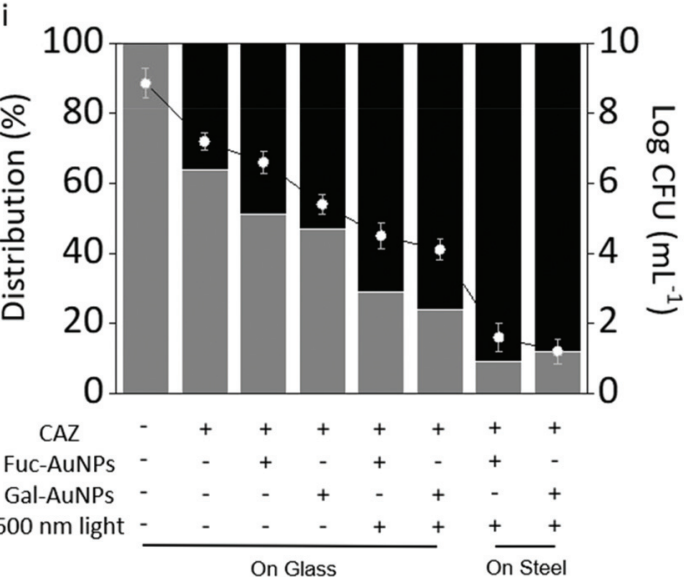

Fig. 5 The evaluation of Fuc-AuNPaCAZ and Gal-AuNP@CAZ for the treatment of biofilms. (a-f) Processed 3D images of $P$. aeruginosa biofilms on a glass-surface using the LIVE/DEAD biofilm viability assay. Dark experiments - (a) untreated (b) Cefazidime (CAZ) $\left(32 \mu \mathrm{g} \mathrm{mL}^{-1}\right.$ ) (c) Fuc-AuNPaCAZ $\left(0.2 \mathrm{nM}\right.$ and $32 \mu \mathrm{g} \mathrm{mL} \mathrm{m}^{-1}$ ) (d) Gal-AuNPaCAZ $(0.2 \mathrm{nM}$ and $32 \mu \mathrm{g} \mathrm{mL}{ }^{-1}$ ) light experiments - (e) Fuc-AuNPaCAZ $(0.2 \mathrm{nM}$ and $32 \mu \mathrm{g} \mathrm{mL}^{-1}$ ) (f) Gal-AuNPaCAZ (0.2 $\mathrm{nM}$ and $32 \mu \mathrm{g} \mathrm{mL}^{-1}$ ) upon $30 \mathrm{~min}$ irradiation of $600 \mathrm{~nm}$ light $\left(1 \mathrm{~W} \mathrm{~cm} \mathrm{~cm}^{-2}\right)$. ( $\mathrm{g}$ and $\mathrm{h}$ ) Processed 3D images of $P$. aeruginosa biofilms on steel surfaces using the LIVE/DEAD biofilm viability assay. (g) Fuc-AuNPaCAZ-treated (0.2 nM and $32 \mu \mathrm{g} \mathrm{mL}^{-1}$ ) and (h) Gal-AuNPaCAZ-treated $\left(0.2 \mathrm{nM}\right.$ and $\left.32 \mu \mathrm{g} \mathrm{mL}{ }^{-1}\right)$ groups upon $30 \mathrm{~min}$ irradiation of $600 \mathrm{~nm}$ light $\left(1 \mathrm{~W} \mathrm{~cm}{ }^{-2}\right)$. (i) Distributions of Pl-stained (black, dead bacteria) and Syto9-stained (grey, live bacteria) $P$. aeruginosa biovolumes and CFU counts (white dots) of all above groups. Biovolume data are mean values of three independent replicates. selective carbohydrate-based recognition of $P$. aeruginosa key virulence factors, LecB/LecA. DCM-loaded Fuc-AuNPs/GalAuNPs afforded a highly selective bacterial fluorescence imaging agent. $^{55}$ While, CAZ loaded Fuc-AuNPs/Gal-AuNPs were shown to have improved therapeutic efficacy towards $P$. aeruginosa when compared to CAZ alone. Exploiting the phototherapeutic properties of the AuNP core (PTT and PDT), light irradiation resulted in an enhanced therapeutic effect towards $P$. aeruginosa when combined with CAZ. Significantly our targeted photo/chemotherapeutics Fuc-AuNP@CAZ/GalAuNP@CAZ were able to eradicate $P$. aeruginosa biofilm formation on glass slides and clinically relevant steel surfaces, with biocompatibility fully confirmed among human cells. Therefore, we believe the ease of preparation (one-pot) and remarkable therapeutic effects observed using this AuNPs strategy, will encourage other groups to develop targeted therapeutic materials in order to combat the important and everworsening problem of antibiotic resistant bacteria.

\section{Conflicts of interest}

There are no conflicts to declare.

\section{Acknowledgements}

The authors thank the NSFC (no. 91853201 and 21907030), the Shanghai Municipal Science and Technology Major Project (2018SHZDZX03), the National Key Sci-Tech Special Projects of Infection Diseases of China (2018ZX10732202), the International Cooperation Program of Shanghai Science and Technology Committee (17520750100) and the China Postdoctoral Science Foundation (2018M630407). TDJ wishes to thank the Royal Society for a Wolfson Research Merit Award.

\section{References}

1 L. R. Mulcahy, V. M. Isabella and K. Lewis, Microb. Ecol., 2014, 68, 1-12.

2 N. M. Maurice, B. Bedi and R. T. Sadikot, Am. J. Respir. Cell Mol. Biol., 2018, 58, 428-439.

3 D. Savoia, Future Microbiol., 2014, 9, 917-928.

4 C. I. Kang, S. H. Kim, H. B. Kim, S. W. Park, Y. J. Choe, M. D. Oh, E. C. Kim and K. W. Choe, Clin. Infect. Dis., 2003, 37, 745-751.

5 O. Ciofu and T. Tolker-Nielsen, Front. Microbiol., 2019, 10, 913, DOI: 10.3389/fmicb.2019.00913.

6 H. C. Flemming, J. Wingender, U. Szewzyk, P. Steinberg, S. A. Rice and S. Kjelleberg, Nat. Rev. Microbiol., 2016, 14, 563-575.

7 D. Nathwani, G. Raman, K. Sulham, M. Gavaghan and V. Menon, Antimicrob. Resist. Infect. Control, 2014, 3, 32-48.

8 V. Aloush, S. Navon-Venezia, Y. Seigman-Igra, S. Cabili and Y. Carmeli, Antimicrob. Agents Chemother., 2006, 50, 43-48. 
9 M. F. Moradali, S. Ghods and B. H. A. Rehm, Front. Cell. Infect. Microbiol., 2017, 7, 1-29.

10 A. Oliver, X. Mulet, C. Lopez-Causape and C. Juan, Drug Resist. Updates, 2015, 21-22, 41-59.

11 X. L. Hu, N. Kwon, K. C. Yan, A. C. Sedgwick, G. R. Chen, X. P. He, T. D. James and J. Yoon, Adv. Funct. Mater., 2020, 30, 1907906.

12 Y. J. Liu, P. Bhattarai, Z. F. Dai and X. Y. Chen, Chem. Soc. Rev., 2019, 48, 2053-2108.

13 X. M. Sun, Z. Liu, K. Welsher, J. T. Robinson, A. Goodwin, S. Zaric and H. J. Dai, Nano Res., 2008, 1, 203-212.

14 B. Hu, L. P. Zhang, X. W. Chen and J. H. Wang, Nanoscale, 2013, 5, 246-252.

15 S. Jeong, J. Lee, B. N. Im, H. Park and K. Na, Biomaterials, 2017, 141, 243-250.

16 Z. H. Yu, X. S. Li, F. G. Xu, X. L. Hu, J. T. Yan, N. Kwon, G. R. Chen, T. T. Tang, X. J. Dong, Y. Y. Mai, D. J. Chen, J. Yoon, X. P. He and H. Tian, Angew. Chem., 2020, 59, 3658-3664.

17 Y. F. Ding, S. K. Li, L. J. Liang, Q. X. Huang, L. H. Yuwen, W. J. Yang, R. B. Wang and L. H. Wang, ACS Appl. Mater. Interfaces, 2018, 10, 9980-9987.

18 Y. H. Feng, L. Liu, J. Zhang, H. Aslan and M. D. Dong, J. Mater. Chem. B, 2017, 5, 8631-8652.

19 J. Zhang, Y. H. Feng, J. L. Mi, Y. T. Shen, Z. G. Tu and L. Liu, J. Hazard. Mater., 2018, 342, 121-130.

20 Q. Y. Chen, L. W. Zhang, Y. H. Feng, F. Shi, Y. B. Wang, P. Wang and L. Liu, J. Mater. Chem. B, 2018, 6, 76437651.

21 J. Li, X. M. Liu, L. Tan, Z. D. Cui, X. J. Yang, Y. Q. Liang, Z. Y. Li, S. L. Zhu, Y. F. Zheng, K. W. K. Yeung, X. B. Wang and S. L. Wu, Nat. Commun., 2019, 10, 4490, DOI: 10.1038/ s41467-019-12429-6.

22 C. Y. Mao, Y. M. Xiang, X. M. Liu, Z. D. Cui, X. J. Yang, Z. Y. Li, S. L. Zhu, Y. F. Zheng, K. W. K. Yeung and S. L. Wu, ACS Nano, 2018, 12, 1747-1759.

23 C. Y. Mao, Y. M. Xiang, X. M. Liu, Z. D. Cui, X. J. Yang, K. W. K. Yeung, H. B. Pan, X. B. Wang, P. K. Chu and S. L. Wu, ACS Nano, 2017, 11, 9010-9021.

24 X. Z. Xie, C. Y. Mao, X. M. Liu, L. Tan, Z. D. Cui, X. J. Yang, S. L. Zhu, Z. Y. Li, X. B. Yuan, Y. F. Zheng, K. W. K. Yeung, P. K. Chu and S. L. Wu, ACS Cent. Sci., 2018, 4, 724738.

25 J. M. Zhang, Y. H. Sun, Y. Zhao, Y. L. Liu, X. H. Yao, B. Tang and R. Q. Hang, Rare Met., 2019, 38, 552-560.

26 C. F. Wang, J. Li, X. M. Liu, Z. D. Cui, D. F. Chen, Z. Y. Li, Y. Q. Liang, S. L. Zhu and S. L. Wu, Biomater. Sci., 2020, 8, 4216-4224.

27 K. Xiong, J. Li, L. Tan, Z. D. Cui, Z. Y. Li, S. L. Wu, Y. Q. Liang, S. L. Zhu and X. M. Liu, Colloid Interface Sci. Commun., 2019, 33, 100201.

28 S. Rana, A. Bajaj, R. Mout and V. M. Rotello, Adv. Drug Delivery Rev., 2012, 64, 200-216.

29 V. Maggi, F. Bianchini, E. Portioli, S. Peppicelli, M. Lulli, D. Bani, R. Del Sole, F. Zanardi, A. Sartori and R. Fiammengo, Chem. - Eur. J., 2018, 24, 12093-12100.
30 D. N. Heo, D. H. Yang, H. J. Moon, J. B. Lee, M. S. Bae, S. C. Lee, W. J. Lee, I. C. Sun and I. K. Kwon, Biomaterials, 2012, 33, 856-866.

31 H. Y. Lee, K. A. Mohammed and N. Nasreen, Am. J. Cancer Res., 2016, 6, 1118-1134.

32 M. F. Attia, N. Anton, J. Wallyn, Z. Omran and T. F. Vandamme, J. Pharm. Pharmacol., 2019, 71, 11851198.

33 S. J. Chadwick, D. Salah, P. M. Livesey, M. Brust and M. Volk, J. Phys. Chem. C, 2016, 120, 10647-10657.

34 M. U. Farooq, V. Novosad, E. A. Rozhkova, H. Wali, A. Ali, A. A. Fateh, P. B. Neogi, A. Neogi and Z. M. Wang, Sci. Rep., 2018, 8, 2907.

35 R. Vankayala, C. C. Lin, P. Kalluru, C. S. Chiang and K. C. Hwang, Biomaterials, 2014, 35, 5527-5538.

36 S. M. Lee, H. J. Kim, Y. J. Ha, Y. N. Park, S. K. Lee, Y. B. Park and K. H. Yoo, ACS Nano, 2013, 7, 50-57.

37 Y. Cheng, A. C. Samia, J. D. Meyers, I. Panagopoulos, B. W. Fei and C. Burda, J. Am. Chem. Soc., 2008, 130, 10643-10647.

38 J. D. Meyers, Y. Cheng, A. M. Broome, R. S. Agnes, M. D. Schluchter, S. Margevicius, X. N. Wang, M. E. Kenney, C. Burda and J. P. Basilion, Part. Part. Syst. Charact., 2015, 32, 448-457.

39 H. B. Mu, J. J. Tang, Q. J. Liu, C. L. Sun, T. T. Wang and J. Y. Duan, Sci. Rep., 2016, 6, 18877, DOI: 10.1038/ srep18877.

40 M. C. Plotkowski, M. Chevillard, D. Pierrot, D. Altemayer, J. M. Zahm, G. Colliot and E. Puchelle, J. Clin. Invest., 1991, 87, 2018-2028.

41 A. V. Grishin, M. S. Krivozubov, A. S. Karyagina and A. L. Gintsburg, Acta Nat., 2015, 7, 29-41.

42 H. Y. Yang, J. J. Zhang, Y. Zang, H. Y. Zhang, J. Li, G. R. Chen and X. P. He, Dyes Pigm., 2017, 136, 224-228.

43 D. M. Richards and R. N. Brogden, Drugs, 1985, 29, 105161.

44 L. Y. Liang and D. Astruc, Coord. Chem. Rev., 2011, 255, 2933-2945.

45 S. Singh, I. S. Dubinsky-Davidchik and R. Kluger, Org. Biomol. Chem., 2016, 14, 10011-10017.

46 J. C. Love, L. A. Estroff, J. K. Kriebel, R. G. Nuzzo and G. M. Whitesides, Chem. Rev., 2005, 105, 11031169.

47 X. P. He, X. L. Hu, H. Y. Jin, J. M. Gan, H. L. Zhu, J. Li, Y. T. Long and H. Tiant, Anal. Chem., 2015, 87, 90789083.

48 T. T. Lou, Y. Q. Wang, J. H. Li, H. L. Peng, H. Xiong and L. X. Chen, Anal. Bioanal. Chem., 2011, 401, 333338.

49 V. Chegel, O. Rachkov, A. Lopatynskyi, S. Ishihara, I. Yanchuk, Y. Nemoto, J. P. Hill and K. Ariga, J. Phys. Chem. C, 2012, 116, 2683-2690.

50 X. Wang, O. Ramstrom and M. D. Yan, J. Mater. Chem., 2009, 19, 8944-8949.

51 P. Arbos, M. Wirth, M. A. Arangoa, F. Gabor and J. M. Irache, J. Controlled Release, 2002, 83, 321-330. 
52 X. L. Hu, L. Y. Chu, X. J. Dong, C. R. Chen, T. T. Tang, D. J. Chen, X. P. He and H. Tian, Adv. Funct. Mater., 2019, 29, 1806986, DOI: 10.1002/adfm.201806986.

53 R. Bresolí-Obach, M. Frattini, S. Abbruzzetti, C. Viappiani, M. Agut and S. Nonell, Sensors, 2020, 20, 5952.
54 R. Vankayala, A. Sagadevan, P. Vijayaraghavan, C. L. Kuo and K. C. Hwang, Angew. Chem., Int. Ed., 2011, 50, 1064010644.

55 W.-T. Dou, Z.-Y. Qin, J. Li, D.-M. Zhou and X.-P. He, Sci. Bull., 2019, 64(24), 1902-1909. 